\title{
Sacrocolpopexia laparoscópica para el tratamiento del prolapso apical: resultados y seguimiento a largo plazo
}

\author{
Claudia López R. ${ }^{1}$, Carolina Cifuentes $P{ }^{2}$, José De Los Ríos $P^{1}{ }^{1}$, Gustavo Calle G. ${ }^{1}$, \\ Juan Castañeda R. ${ }^{1}$. Luis Almanza P. ${ }^{1}$, Ricardo Vásquez $R .{ }^{1}$, Adriana Arango M. ${ }^{1}$, Luis \\ Jiménez $Z^{1}$ \\ ${ }^{1}$ Unidad de Endoscopia Ginecológica, Clínica del Prado, ${ }^{2}$ Programa de Cirugía Endoscópica Ginecológica. Universidad \\ CES, Medellín, Colombia.
}

\section{RESUMEN}

Objetivos: Evaluar los resultados subjetivos, anatómicos y funcionales a largo plazo de las pacientes sometidas a sacrocolpopexia laparoscópica para manejo de prolapso apical. Métodos: Estudio observacional con pacientes a quienes se les hizo sacrocolpopexia laparoscópica entre febrero de 2006 y diciembre de 2012, en tres centros. El nivel de soporte del piso pélvico se midió mediante la escala de cuantificación del prolapso de órganos pélvicos (POP-Q). Los resultados funcionales se evaluaron mediante un cuestionario de síntomas intestinales, urinarios, sexuales y de molestias físicas. También se estimó la satisfacción global de las pacientes con una escala de uno a diez. Resultados: Se realizó sacrocolpopexia laparoscópica a 68 pacientes, pero el seguimiento fue posible sólo en 24 . Ninguna paciente tuvo prolapso apical postoperatorio. El punto $C$ medio del $P O P-Q$ fue $-6,8 \mathrm{~cm}$. Se observó mejoría importante con respecto a los síntomas subjetivos de prolapso con reducciones significativas en las puntuaciones del cuestionario en el seguimiento postoperatorio. La satisfacción fue en promedio de 9,1. La incontinencia urinaria preoperatoria se resolvió en el $35 \%$ de las que la reportaron, sin necesidad de cirugía de continencia concomitante. No hubo complicaciones a corto plazo. A largo plazo hubo una hernia incisional en el sitio del trocar y una obstrucción intestinal por la malla. Conclusión: La sacrocolpopexia laparoscópica es un tratamiento quirúrgico seguro y eficaz para el prolapso apical post-histerectomía. Proporciona un excelente soporte apical y buen nivel de satisfacción, con una mejoría general de los síntomas de prolapso.

\section{PALABRAS CLAVE: Sacrocolpopexia, laparoscopia, prolapso de órganos pélvicos, prolapso de cúpula vaginal}

\section{SUMMARY}

Objective: To evaluate the long-term subjective, anatomical and functional outcomes after laparoscopic sacrocolpopexy for apical prolapse. Methods: An observational study of women undergoing laparoscopic sacrocolpopexy between February 2006 and December 2012 was undertaken, at three centers. Pelvic organ support was assessed objectively using the pelvic organ prolapse quantification scale (POP-Q). Functional outcomes were assessed using a questionnaire of bowel, urinary, sexual and physical discomfort symptoms postoperatively. Also was assessed the overall satisfaction of surgery with a scale of one to ten. Results: During the period of the study, sacrocolpopexy was done in 68 patients, but follow-up was possible only in 24. At a mean follow up of 34 months, all 24 women had stage 0 vault support with point $C$ of the POP-Q score averaging $-6.8 \mathrm{~cm}$. Subjective improvements in prolapse symptoms were observed with significant reductions in the questionnaire scores. The satisfaction measured with visual scale averaged 9.1. Fifteen women 
reported stress urinary incontinence before sacrocolpopexy, and it was resolved in 35\% without concomitant continence surgery. New onset incontinence was reported in two women. There were no intraoperative and perioperative complications. The long term complications were an incisional hernia on the trocar port site and bowel obstruction caused by the mesh that needed intestinal resection. Conclusions: Laparoscopic sacrocolpopexy is a safe and effective surgical treatment for post-hysterectomy apical prolapse. It provides excellent apical support and good level of satisfaction, with overall improvement in prolapse symptoms.

\section{KEY WORDS: Sacrocolpopexy, laparoscopy, pelvic organ prolapsed, vaginal vault prolapse}

\section{INTRODUCCIÓN}

El prolapso del ápex vaginal después de histerectomía es una patología con impacto negativo en la calidad de vida de las mujeres, debido a su asociación con disfunción sexual, anorrectal y urinaria. Tiene una incidencia desconocida, sin embargo, en la literatura se ha estimado que se encuentra entre 0,36 a 3,6 por 1.000 mujeres/año, con una incidencia acumulada del $0,5 \%(1)$.

Si bien se han descrito varias técnicas para la corrección del prolapso apical, la sacrocolpopexia abdominal, se ha considerado el estándar de oro, con tasas de éxito reportadas en la literatura entre 70 y $100 \%$. Cuando se le compara con la fijación al ligamento sacroespinoso por vía vaginal, se observan menores tasas de recurrencia y de dispareunia postoperatorias para la sacrocolpopexia (2).

El abordaje laparoscópico fue descrito por primera vez en 1994 por Nezhat y cols (3). La literatura dispone de un solo ensayo clínico aleatorizado que compara la técnica laparoscópica con la sacrocolpopexia por laparotomía y que demuestra equivalencia clínica entre los dos procedimientos al año de seguimiento (4). No se cuenta aún con datos de seguimiento de estas pacientes después de un año de la intervención.

El objetivo de nuestro estudio fue evaluar a largo plazo los resultados anatómicos, funcionales y de satisfacción, en las pacientes sometidas a sacrocolpopexia laparoscópica para el tratamiento del prolapso apical post-histerectomía.

\section{PACIENTES Y MÉTODOS}

Selección de pacientes: Se revisaron los registros de sacrocolpopexias laparoscópicas realizadas entre febrero de 2006 y diciembre de 2012 en tres centros de Medellín, Colombia (Clínica del Prado, Clínica Medellín y Clínica Profamilia). Se examinaron los archivos clínicos en su totalidad para consignar los datos de la evaluación preoperatoria, la cual incluía historia médica detallada, edad, cirugías urológicas y ginecológicas previas, antecedentes obstétricos y aparición de menopausia. Del examen físico se tomaron los datos del índice de masa corporal y del tipo y grado de prolapso de acuerdo con la cla- sificación de Baden-Walker que se utilizaba en ese entonces. Respecto al procedimiento quirúrgico, se registraron el tiempo operatorio, la pérdida sanguínea y las complicaciones intra y postoperatorias y el tiempo de hospitalización en el postoperatorio.

Procedimiento: La técnica de sacrocolpopexia utilizada, fue la propuesta por Wattiez y cols (5). La intervención fue practicada posterior a profilaxis antibiótica, con cateter vesical y bajo anestesia general. Se introdujo un separador maleable en la cúpula vaginal. Después de la creación del neumoperitoneo, se pasó endoscopio a través de puerto umbilical de $10 \mathrm{~mm}$ y se insertaron 3 trocares auxiliares de $5 \mathrm{~mm}$, dos pararrectales izquierdos, en flanco y fosa ilíaca respectivamente y otro pararrectal derecho en la fosa ilíaca. En posición de Trendelemburg, se disecó el promontorio del sacro hasta localizar el ligamento longitudinal anterior, y posteriormente se incidió el peritoneo parietal desde el promontorio hasta el saco de Douglas en la pelvis menor en un trayecto medial al uréter y uterosacro derechos y lateral al meso-recto. Posteriormente, se disecó el peritoneo que recubría el ápex vaginal y se realizó disección sobre la superficie anterior de la vagina hasta localizar claramente la fascia pubocervical y en la cara posterior, hasta encontrar la fascia rectovaginal y los músculos elevadores del ano a lado y lado del recto. Hecha la disección se procedió a fijar un recuadro de malla de polipropileno (Gynemesh, Ethicon Inc) (6), macroporo y monofilamento de aproximadamente $3 \times 3 \mathrm{~cm}$ a la porción disecada de la fascia pubocervical con 4 a 6 puntos de poliéster calibre cero (Ethibond, Ethicon Inc). Un fragmento de dicha malla en forma de "h" de $12 \times 4 \mathrm{~cm}$, cuyos extremos inferiores fueron fijados cada uno a los músculos elevadores del ano a cada lado; fueron usados 4 a 6 puntos para anclarla a la fascia rectovaginal en la cara posterior de la vagina y finalmente, el extremo superior de dicha malla fue fijado con uno o dos puntos al ligamento longitudinal anterior del sacro tras asegurarse de que la malla tenía la tensión suficiente para corregir el prolapso sin quedar con exceso de tensión. Por último, se cerró el peritoneo disecado con una sutura continua de poliglactina 2.0 (Vicryl, Ethicon Inc) recubriendo la totalidad de la malla para dejarla en posición retroperitoneal. 
Evaluación postoperatoria. Posterior a la recopilación de la información se creó una base de datos y se contactó telefónicamente a las pacientes para que asistieran a una cita de control. En dicha visita médica de seguimiento se evaluaron los diferentes desenlaces:

1. Cuestionario sobre síntomas actuales: Se realizó un cuestionario para medir el éxito quirúrgico subjetivo en todas las pacientes. Las variables evaluadas incluían síntomas urinarios, digestivos y de función sexual entre otros, antes y después de la cirugía

2. Examen físico y medición del POP-Q: La tasa de recurrencia de prolapso fue evaluada mediante examen físico con el sistema POP-Q. La principal medida objetiva para considerar un resultado como exitoso fue la ausencia de prolapso de cúpula vaginal postoperatoria teniendo en cuenta la medición del punto $C$. También se registró la presencia de prolapso en otro compartimiento.

3. Se actualizaron datos de seguimiento y se interrogó sobre necesidad de cirugías posteriores a la sacrocolpopexia.

4. Se solicitó cuantificar el grado de satisfacción con la cirugía realizada. La satisfacción con respecto a la cirugía fue evaluada por medio de una escala de uno a diez.

\section{RESULTADOS}

Después de revisar las historias clínicas en su totalidad, se encontraron 68 pacientes que se sometieron a sacrocolpopexia laparoscópica, de estas sólo fue posible contactar a 24 mujeres para que asistieran a seguimiento.

Datos demográficos. Veinticuatro mujeres, con una edad media de 63 años fueron incluidas. En el grupo de estudio, la paridad media fue de 4,3 con promedio de partos vaginales de 3,5 y cesáreas 0,04 . Las características demográficas preoperatorias de la población de estudio se muestran en la Tabla I.

Datos del procedimiento quirúrgico. Todos los

Tabla I

VARIABLES DEMOGRÁFICAS

\begin{tabular}{lc}
\hline Variable & Promedio (rango) \\
\hline Edad & $62,7(54-81)$ \\
Número de embarazos & $4,3(1-14)$ \\
Número de partos vaginales & $3,5(1-12)$ \\
Número de cesáreas & $0(0-1)$ \\
Peso & $62,3(45-87)$ \\
IMC & $25,6(18-37)$ \\
\hline
\end{tabular}

IMC: Índice de masa corporal. procedimientos fueron completados por via laparoscópica, ninguna paciente requirió laparoconversión. En cuanto a las cirugías ginecológicas previas a la sacrocolpopexia laparosópica, a 11 mujeres (46\%) se les había realizado anteriormente una intervención quirúrgica para corrección de prolapso (histerectomía vaginal más corrección de celes: $30 \%$; colporrafia anterior y posterior: $13 \%$; sacrocolpopexia laparoscópica: $4 \%$ ) y a 9 pacientes (38\%) alguna cirugía para incontinencia urinaria (TVT: $4 \%$; Burch: $33 \%$ ). El tipo de histerectomía previa a la sacrocolpopexia laparoscópica fueron: abdominal $63 \%$, vaginal $29 \%$ y laparoscópica $8 \%$. A 13 mujeres $(54,2 \%)$ se les realizaron procedimientos adicionales a la sacrocolpopexia y a 11 sacrocolpopexias puras $(45,8 \%)$. A 18 pacientes (75\%) se les colocó malla Gynemeh PS y a las 6 restantes de Prolene (25\%); 33\% de las pacientes fueron manejadas ambulatoriamente en el postoperatorio, el resto tuvo una estancia hospitalaria de 1 día. Las variables del procedimiento quirúrgico se describen en la Tabla II.

Cuestionario. El tiempo de seguimiento de las pacientes fue en promedio de 34 meses. Las 24 mujeres que estaban disponibles para el seguimiento completaron el cuestionario de percepción subjetiva de los síntomas postoperatorios y de satisfacción. El grado de satisfacción después de la cirugía medido con escala de uno a diez fue en promedio de 9,1 (rango: 1 a 10). De las 24 pacientes que reportaron sensación de masa genital previa al procedimiento, el $87,5 \%$ experimentaron mejoría total y $4 \%$ refirieron mejoría parcial, con cura subjetiva de $91,5 \%$. La evaluación de los síntomas respecto a lo registrado previo a la cirugía se observa en la Tabla III. En 15 pacientes refirieron incontinencia urinaria de esfuerzo antes de la intervención, y después de la cirugía 35\% manifestaron mejoría total y $29 \%$ parcial; de estas pacientes, solo una tuvo cirugía de continencia concomitante. Dos pacientes experimentaron incontinencia urinaria de novo. Los síntomas de novo que aparecieron después de la cirugía se presentan en Tabla IV.

\section{Tabla II \\ VARIABLES DEL PROCEDIMIENTO QUIRÚRGICO REALIZADO}

\begin{tabular}{lc}
\hline Variable & Promedio (rango) \\
\hline Tiempo quirúrgico (minutos) & $150(105-260)$ \\
Pérdida sanguínea (ml) & $17(5-50)$ \\
Días de hospitalización & $0,6(0-1)$ \\
Satisfacción resultado & $9,1(1-10$ \\
quirúrgico (EVA 1-10) &
\end{tabular}

EVA: escala visual análoga. 
Tabla III

EVOLUCIÓN DE LOS SÍNTOMAS DESPUÉS DE LA CIRUGÍA

\begin{tabular}{lccccc}
\hline Síntoma & $\begin{array}{c}\text { Antes de la cirugía } \\
\text { Con síntomas } \\
\mathrm{n}(\%)\end{array}$ & $\begin{array}{c}\text { Mejoría total } \\
\mathrm{n}(\%)\end{array}$ & $\begin{array}{c}\text { Mejoría parcial de cirugía } \\
\mathrm{n}(\%)\end{array}$ & $\begin{array}{c}\text { Sin cambios } \\
\mathrm{n}(\%)\end{array}$ & $\begin{array}{c}\text { Peor } \\
\mathrm{n}(\%)\end{array}$ \\
\hline Peso pélvico & $17(70,8)$ & $14(82)$ & $2(12)$ & $1(6)$ & 0 \\
Sensación de masa & $24(100)$ & $21(87,5)$ & $1(4)$ & $2(8)$ & 0 \\
Dificultad para orinar & $8(33)$ & $8(100)$ & 0 & 0 & 0 \\
IU esfuerzo & $17(70)$ & $6(35)$ & $5(29)$ & $3(17,6)$ & $3(17,6)$ \\
IU urgencia & $15(62,5)$ & $3(20)$ & $1(6,6)$ & $9(60)$ & $2(13,3)$ \\
Constipación & $10(41,6)$ & 0 & 0 & $7(70)$ & $3(30)$ \\
I fecal & $3(12,5)$ & $1(33)$ & 0 & 0 & $2(66)$ \\
Dispareunia & $3(12,5)$ & $1(33)$ & 0 & $2(66)$ & 0 \\
Lumbalgia & $2(8,3)$ & 0 & 0 & $2(100)$ & 0 \\
ITU a repetición & $10(41,6)$ & $2(20)$ & $3(30)$ & $4(40)$ & $1(10)$ \\
\hline
\end{tabular}

IU: incontinencia urinaria. ITU: infección del tracto urinario. I: incontinencia.

Tabla IV

SÍNTOMAS DE NOVO DESPUÉS DE LA CIRUGÍA

\begin{tabular}{lcc}
\hline Síntomas & Antes & \multicolumn{2}{c}{$\begin{array}{c}\text { Después } \\
\text { Sin síntoma antes de la cirugía } \\
\mathrm{n}(\%)\end{array}$} & $\begin{array}{c}\text { Con síntomas después de la cirugía } \\
\mathrm{n}(\%)\end{array}$ \\
\hline Incontinencia urinaria de esfuerzo & $7(29,1)$ & $2(28)$ \\
Incontinencia urinaria de urgencia & $9(37,5)$ & $3(33)$ \\
Incontinencia fecal & $21(87,5)$ & $2(9,5)$ \\
Dispareunia & $13(54,1)$ & $2(15,3)$
\end{tabular}

Evaluación postoperatoria del prolapso (medición POP-Q). Antes de la intervención, la mayoría de mujeres tenían un descenso significativo de la cúpula vaginal (Tabla V). En la visita de seguimiento se realizó medición de acuerdo al sistema POP$\mathrm{Q}$ a todas las pacientes. La medición del punto $\mathrm{C}$ fue en promedio de $-6,8$. Ninguna mujer presentó prolapso apical después de la intervención. Lo cual significa una cura objetiva del $100 \%$. Los valores medios de los puntos $\mathrm{Aa}, \mathrm{Ba}, \mathrm{Ap}$ y $\mathrm{Bp}$, fueron $-2,5$, $-2,4,-2,7$ y $-2,6$ que en promedio hablaría de un prolapso anterior o posterior estadio I (Tabla VI). No se presentaron recurrencias de prolapso apical.

Intervenciones posteriores. Una paciente al año de la sacrocolpopexia requirió colporrafia anterior y posterior por cistorrectoenterocele. A 3 pacientes, después de la intervención, se les realizó cabestrillo suburetral por incontinencia urinaria de esfuerzo.

Complicaciones. No se observaron complicaciones intraoperatorias ni en el postoperatorio inmediato. A largo plazo, se presentaron una hernia
Tabla V

\section{GRADO DE PROLAPSO ANTES DE LA CIRUGÍA SEGÚN CLASIFICACIÓN BADEN-WALKER}

\begin{tabular}{cc}
\hline Grado de prolapso & $\mathrm{n}(\%)$ \\
\hline 1 & $1(4)$ \\
2 & $1(4)$ \\
3 & $20(84)$ \\
4 & $2(8)$ \\
\hline
\end{tabular}

Tabla VI

\section{MEDIDAS POP-Q POSOPERATORIAS}

\begin{tabular}{ccccc}
\hline \multicolumn{5}{c}{ POP-Q } \\
Aa & Ba & C & Ap & Bp \\
\hline$-2,5$ & $-2,4$ & $-6,8$ & $-2,7$ & 2,6 \\
R: 1 a -3 & R: 1 a -3 & R: -5 a -9 & R: -2 a -3 & R: -2 a -3
\end{tabular}

$R$ rango. 
en el sitio del trocar y una obstrucción intestinal ocasionada por la malla, que requirió resección, tres años después de la cirugía.

\section{DISCUSIÓN}

La sacrocolpopexia es el tratamiento quirúrgico de elección recomendado para el manejo del prolapso de cúpula vaginal, como se demostró al compararlo con la fijación al ligamento sacroespinoso por vía vaginal (2). Se ha demostrado equivalencia clínica al año de seguimiento, entre la vía laparoscópica y la vía abdominal en un ensayo clínico aleatorizado (4).

Muchos autores han publicado su experiencia con la sacrocolpopexia laparoscópica para el tratamiento de prolapso de cúpula posthisterectomía, concluyendo que es un tratamiento quirúrgico seguro y eficaz, que proporciona un excelente soporte apical (7-16). Las tasas de curación objetiva para sacrocolpopexia laparoscópica publicadas por la literatura son del $75 \%$ al $100 \%$, con tasas de curación subjetiva que van desde $89 \%$ a $98 \%$ (17).

Nuestro estudio, que tuvo un tiempo de seguimiento en promedio de 34 meses, apoya los resultados de los trabajos previos, y demuestra que la sacrocolpopexia laparoscópica es una técnica fiable y útil, que ofrece un excelente soporte apical confirmado por un punto $C$ del POP-Q en $-6,8$. El resultado para el soporte anterior y posterior fue menos predecible. Los valores medios de los puntos $\mathrm{Aa}, \mathrm{Ba}, \mathrm{Ap}$ y $\mathrm{Bp}$ fueron en promedio correspondientes a un prolapso anterior o posterior estadio I.

Es de destacar la escasa pérdida sanguínea de $17 \mathrm{ml}$ (rango: 5 y $50 \mathrm{ml}$ ), dentro de los límites reportados en otros estudios (4). El tiempo quirúrgico fue 157 minutos en promedio. Los tiempos operatorios para la sacrocolpopexia laparoscópica informados por la literatura se encuentran entre 97-269 minutos, que por supuesto están sujetos a los diferentes niveles de la experiencia del cirujano, y a los procedimientos concomitantes realizados $(17,18)$. El tiempo de estancia hospitalaria en nuestra experiencia fue corto de 0,6 días (rango: 0 a 1), lo cual deriva en menor riesgo de complicaciones secundarias al reposo prolongado en cama en el postoperatorio y en menores costos. Otros reportes en la literatura muestran estancias hospitalarias mayores de en promedio dos días (4), así que nuestros resultados apoyan un tiempo menor de hospitalización sin efectos adversos en las pacientes.

Por otro lado, los síntomas de prolapso tuvieron una mejoría global acorde con lo que muestran otros trabajos en sus cuestionarios de calidad de vida (11-12). Como se mencionó, de las 15 pacientes que refirieron incontinencia urinaria de esfuerzo antes de la intervención, 35\% manifestaron mejoría total y $29 \%$ parcial, después de la cirugía. De estas pacientes, solo una tuvo cirugía de continencia urinaria concomitante. Un estudio prospectivo previo al nuestro, evidenció resolución de la incontinencia urinaria en la mitad de sus pacientes sin necesidad de cirugía de continencia concomitante (11). Mientras que otro estudio retrospecivo de cohortes que evaluó la incidencia de incontinencia después de sacrocolpopexia laparoscópica en mujeres con pruebas preoperatorias urodinámicas de incontinencia de estrés con reducción del prolapso negativas, observó que después de al menos 1 año de seguimiento, aproximadamente una de cada seis mujeres finalmente se sometieron a una cirugía de cabestrillo (19). La realización de procedimientos de continencia de rutina concomitantes, en el momento de sacrocolpopexia laparoscópica sigue siendo un área de controversia, los estudios aleatorizados en sacrocolpopexia abdominal han obtenido resultados contradictorios (20-21). Hacen falta estudios aleatorizados en sacrocolpopexia laparoscópica con respecto a la cirugía de incontinencia profiláctica.

Una revisión realizada por Ganatra y cols (18), mostró en los estudios analizados, un nivel medio de satisfacción de las pacientes de 94,4\%. En nuestro trabajo, el grado de satisfacción también fue aceptable y fue en promedio de 9,1 sobre 10 .

En los estudios disponibles la recurrencia de prolapso apical se presentó en un rango entre $0 \mathrm{y}$ $44 \%$ (18). En nuestra experiencia no se presentaron recurrencias de prolapso del ápex vaginal. En la revisión de Ganatra y cols (18), se presentó disfunción intestinal postoperatoria, incluyendo estreñimiento, dolor anal e incontinencia fecal en el 9,8\% de las pacientes (rango: 0-25\%). En nuestros resultados, el 9,5\% de las pacientes presentó incontinencia fecal de novo. En varios estudios $(12,18)$, la función sexual postoperatoria fue evaluada con un $8 \%(0-47 \%)$ de las pacientes tenían disfunción sexual después de sacrocolpopexia laparoscópica. En nuestro estudio, 13 pacientes tenían vida sexual activa, y de estas 2 (15\%) refirieron aparición de dispareunia posterior a la cirugía. Diferentes estudios han evaluado la función sexual postoperatoria, encontrando en el $8 \%$ de las pacientes (rango: $0-47 \%$ ) disfunción sexual después de la sacrocolpopexia laparoscópica $(12,18)$.

Es necesario un período de seguimiento más largo para sacar conclusiones más firmes con respecto a la tasa de prolapso recurrente y a las complicaciones asociadas a la malla. 


\section{CONCLUSIÓN}

La sacrocolpopexia laparoscópica para el tratamiento del prolapso apical después de histerectomía, ofrece buenos resultados objetivos a largo plazo, con adecuado nivel de satisfacción, baja recurrencia, poca morbilidad y calidad de vida aceptable.

\section{REFERENCIAS}

1. Dällenbach P, Kaelin-Gambirasio I, Jacob S, Dubuisson JB, Boulvain M. Incidence rate and risk factors for vaginal vault prolapse repair after hysterectomy. Int Urogynecol J Pelvic Floor Dysfunct 2008;19:1623-9.

2. Maher C, Feiner B, Baessler K, Schmid C. Surgical management of pelvic organ prolapse in women. Cochrane Database Syst Rev 2013 Apr 30;4:CD004014.

3. Nezhat CH, Nezhat F, Nezhat C. Laparoscopic sacral colpopexy for vaginal vault prolapse. Obstet Gynecol 1994;84:885-8.

4. Freeman RM, Pantazis K, Thomson A, Frappell J, Bombieri L, Moran P, Slack M, Scott P, Waterfield M . A randomised controlled trial of abdominal versus laparoscopic sacrocolpopexy for the treatment of posthysterectomy vaginal vault prolapse: LAS study. Int Urogynecol J 2013;24:377-84.

5. Wattiez A, Canis M, Mage G, Pouly JL, Bruhat MA. Promontofixation for the treatment of prolapse. Urol Clin North Am 2001;28:151-7.

6. Agarwala N, Hasiak N, Shade M. Laparoscopic sacral colpopexy with Gynemesh as graft material: experience and results. J Minim Invasive Gynecol 2007;14:577-83.

7. Sundaram CP, Venkatesh R, Landman J, Klutke CG. Laparoscopic sacrocolpopexy for the correction of vaginal vault prolapse. J Endourol 2004;18:620-3.

8. Ross JW, Preston M. Laparoscopic sacrocolpopexy for severe vaginal vault prolapse: 5 -year outcome. J Minim Invasive Gynecol 2005;12:221-6.

9. Cosson M, Rajabally R, Bogaert E, Querleu D, Crépin G. Laparoscopic sacrocolpopexy, hysterectomy, and Burch colposuspension: feasibility and short-term complications of 77 procedures. JSLS 2002;6:115-9
10. Higgs PJ, Chua HL, Smith AR. Long term review of laparoscopic sacrocolpopexy. BJOG 2005;112:1134-8.

11. North C, Ali-Ross N, Smith A, Reid F. A prospective study of laparoscopic sacrocolpopexy for the management of pelvic organ prolapse. BJOG 2009;116:1251-7.

12. Price N, Slack A, Jackson SR. Laparoscopic sacrocolpopexy: an observational study of functional and anatomical outcomes. Int Urogynecol J Pelvic Floor Dysfunct 2011;22:77-82.

13. Chan CM, Liang HH, Go WW, To WW, Mok KM. Laparoscopic sacrocolpopexy for uterine and post-hysterectomy prolapse: anatomical and functional outcomes. Hong Kong Med J 2011;17:301-5.

14. Bojahr B, Tchartchian G, Waldschmidt M, Ohlinger R, De Wilde RL. Laparoscopic sacropexy: a retrospective analysis of the subjective outcome in 310 cases. Obstet Gynecol Int 2012;2012:538426.

15. Granese R, Candiani M, Perino A, Romano F, Cucinella G. Laparoscopic sacrocolpopexy in the treatment of vaginal vault prolapse: 8 years experience. Eur $\mathrm{J}$ Obstet Gynecol Reprod Biol 2009;146:227-31.

16. Sabbagh R, Mandron E, Piussan J, Brychaert PE, Tu le $M$. Long-term anatomical and functional results of laparoscopic promontofixation for pelvic organ prolapsed. BJU Int 2010;106:861-6.

17. Frick AC, Paraiso MF. Laparoscopic management of incontinence and pelvic organ prolapse. Clin Obstet Gynecol 2009;52:390-400

18. Ganatra AM, Rozet F, Sanchez-Salas R, Barret E, Galiano M, Cathelineau X, Vallancien G. The current status of laparoscopic sacrocolpopexy: a review. Eur Urol 2009;55:1089-103.

19. Leruth J, Fillet M, Waltregny D. Incidence and risk factors of postoperative stress urinary incontinence following laparoscopic sacrocolpopexy in patients with negative preoperative prolapse reduction stress testing . Int Urogynecol J 2013;24:485-91.

20. Costantini E, Zucchi A, Giannantoni A, Mearini L, Bini V, Porena M. Must colposuspension be associated with sacropexy to prevent postoperative urinary incontinence? Eur Urol 2007;51:788-94.

21. Brubaker L, Cundiff GW, Fine P, Nygaard I, Richter $\mathrm{HE}$, Visco AG, et al. Abdominal sacrocolpopexy with Burch colposuspension to reduce urinary stress incontinence. N Engl J Med 2006;354:1557-66. 